\title{
Medición de niveles de ácido acético en el proceso de limpieza de máquinas hemodialíticas en una unidad de tratamiento renal.
}

Measurement of the levels of acetic acid in the process of cleaning of machines hemodialiticas in a renal treatment unit

Cristian Laverde Albarracín. ${ }^{1}$, Ruth Isabel Torres. ${ }^{2}$, Jean Carlos Simancas Vargas. ${ }^{3}$, Adriano Perez Toapanta. ${ }^{4} \&$ Cristian Fernando Guaman Sanchez. ${ }^{5}$

\begin{abstract}
.
DOI: https://doi.org/10.33262/cienciadigital.v2i3.153

The chemical risks not controlled generally concur in important affectations to the health of the workers in the different processes that these are. The present study was conducted in a private renal treatment unit, where hemodialysis therapies are practiced to treat patients with kidney failure. To ensure that this therapy is adequate several processes are carried out, among which is the descaling of hemodialysis machines, where the use of chemicals such as acetic acid is necessary to disinfect the equipment indicated. For this, the concentration levels of this acid were measured with the absorption method in carbon and ion chromatography, to then compare them with the Threshold Limit Values TLV.

After analyzing the results obtained, it is concluded that the concentration of acetic acid in the transfer process in the case of TLV-STEL exceeds the permitted concentration.

\footnotetext{
${ }^{1}$ Universidad UTE \& Universidad Técnica Estatal de Quevedo, Santo Domingo de los Tsachilas, Los Ríos, Ecuador, cristian.laverde@ute.edu.ec; claverde@uteq.edu.ec

2Universidad Técnica Estatal de Quevedo, Los Ríos, Ecuador, ritorres@uteq.edu.ec

3 Universidad Técnica Estatal de Quevedo, Los Ríos, Ecuador, jsimancas@uteq.edu.ec

${ }^{4}$ Universidad Técnica Estatal de Quevedo, Los Ríos, Ecuador, aperez@uteq.edu.ec ${ }^{5}$ Universidad de Cuenca, Cuenca, Ecuador, cristian.guaman@ucuenca.edu.ec
} 
Finally, general measures are established, tending to control the effects of this result to guarantee the people involved the development of their activities in a safe environment.

Keywords: Riesgo Químico, TLV-TWA. TLV-STEEL, Desincrustación, Medición.

\section{Resumen.}

Los riesgos químicos no controlados generalmente concurren en afectaciones importantes a la salud de los trabajadores en los diferentes procesos que estos se encuentran. El presente estudio se realizó en una unidad privada de tratamiento renal, donde se practican terapias de hemodiálisis para tratar a pacientes enfermos con insuficiencia renal. Para asegurar que esta terapia sea adecuada se realizan varios procesos dentro de los cuales se encuentra la desincrustación de máquinas de hemodiálisis, en donde es necesario el uso de químicos como el ácido acético que permite la desinfección de los equipos señalados. Para esto se midieron los niveles de concentración de este ácido con el método de absorción en carbón y cromatografía iónica, para luego compararlos con los Valores Umbrales Límite TLV.

Luego del análisis de resultados obtenidos, se concluye que la concentración del ácido acético en el proceso de trasvase en el caso del TLV- STEL excede la concentración permitida.

Finalmente se establecen medidas generales, tendientes a controlar los efectos de este resultado para garantizar a las personas involucradas el desarrollo sus actividades en un ambiente seguro.

Palabras Claves: Riesgo Químico, TLV-TWA. TLV-STEEL, Desincrustación, Medición.

\section{Introducción .}

En el mundo se han identificado más de 25 millones de sustancias químicas, de las cuales se comercializan y están registradas 100.195 en la Unión Europea. Las empresas han usado hasta 30.000 de estos productos, aunque a unos 20.000 no se les ha realizado pruebas toxicológicas y el $21 \%$ de las sustancias de mayor demanda en la producción carecen de información toxicológica (Calera 2005).

Recientes estudios señalan que cada año en países como España más de 4000 trabajadores mueren por enfermedades derivadas de la exposición a sustancias químicas, y más de 33000 enferman debido a la misma razón. Lamentablemente nuestro país todavía no cuenta con estadísticas que permitan entender la incidencia de este tipo de riesgo sobre la salud de los trabajadores (Asturias 2000) 
Sin embargo de ello y frente a los datos internacionales citados, se concluye que medir y establecer medidas de prevención frente a los riesgos químicos es menester sobre todo en empresas cuyas actividades demandan el contacto permanente con sustancias químicas. (Castro 1998).

Las primeras listas de concentraciones máximas en el ambiente laboral fueron establecidas entre 1933 y 1938 en la Unión Soviética, Suecia y los Estados Unidos. La Unión Soviética fue el primer país en establecer una regulación obligatoria (OIT, 1983). En los Estados Unidos estas listas fueron originalmente llamadas Concentraciones Máximas Permisibles y posteriormente asumieron la denominación de Threshold Limit Values (TLV)1 (Jeffrey 1984; Paull, 1984; OIT, 1983; Breyse, 1991). La lista de los TLVs creció rápidamente y ha sido utilizada ampliamente en los países occidentales (Henschler, 1984; Paustenbach and Langner, 1986). A partir de la década de los 60, diferentes países han venido desarrollando sus propias listas, como es el caso de Alemania a partir de 1968 y los Países Nórdicos a finales de la década de los 70. Por su parte, la Unión Soviética desarrolló sus propios valores límites desde la década de los 30 los cuales, es importante señalar, se basan en conceptos completamente diferentes a los utilizados tradicionalmente en los Estados Unidos y Europa Occidental (Izmerov, 1973). Holanda y Suecia han desarrollado sus propias listas sobre las base de una progresista filosofía social y de validación científica. A pesar de los esfuerzos por lograr una unificación de criterios a nivel internacional promovidos por la OMS, la OIT y la CEE durante los años 1980 y 1981 todos han fracasado por diversas razones, principalmente por las diferencias entre las bases y criterios científicos para el establecimiento de los límites de exposición u Occupational Expossure Limits (OELs)2 y las condiciones industriales de los diferentes países (Yanes, 2002)

El estudio se realizó en una Unidad Privada de Tratamiento Renal en Quito- Ecuador, donde el uso de ácido acético para la limpieza de las máquinas de hemodiálisis es permanente, por lo cual los trabajadores encargados de esta actividad tienen contacto y exposición ante este tipo de riesgo.

En el casos, se midieron en el lugar de trabajo niveles de ácido acético que superan en gran medida los umbrales recomendados (Paloucci, 2015).

Con esta información se podrán dirigir los esfuerzos hacia la protección del personal expuesto proponiendo mejoras en organización del trabajo, equipos, planes de contingencia, entre otros. Teniendo en cuenta lo anterior, se hace necesario realizar estudios a fin de evitar las consecuencias que puede tener la exposición al ácido acético (CujarVertel 2016).

Los resultados obtenidos permitirán generar información (inexistente en el momento) para que organizaciones que tienen el mismo giro de negocio puedan tomar las medidas de prevención necesarias, y proteger a sus colaboradores de la exposición al ácido acético. 


\section{Objetivo.}

Los trabajadores de la Unidad Privada Renal, están expuestos a diferentes riesgos, el proceso que se realiza con mayor periodicidad y frecuencia es la limpieza de las máquinas de hemodiálisis de allí el interés de centrar la investigación en el nivel de exposición al riesgo químico al ácido acético para evitar el efecto nocivo del mismo, las conclusiones de la investigación, permitirán tomar medidas para mejorar las condiciones laborales de los trabajadores en exposición al agente químico en estudio.

Para prevenir el riesgo químico, lo primero que se debe hacer es informarse sobre el compuesto químico que se va a manejar (Cabañas, 2001), por lo que la información presentada en la investigación, es de suma importancia, para la gestión de la prevención.

\section{Materiales y métodos.}

El método de Higiene Industrial usado para la medición del ácido acético en el puesto de Trabajo propuesto en la investigación, es el de Absorción en carbón/Cromatógrafía Ionica y la comparación de los valores con la tabla de los TLVs, usando los criterios de valoración propuestos por la ACGIH (American Conference of Governmental Industrial Hygienists), que son los límites de exposición o de concentración de sustancias en la atmósfera en los lugares de trabajo, ponderadas en el tiempo y calculadas para una exposición de ocho horas por día y 40 horas por semana (Mangosio, 2008).

Debido a los variados efectos que las sustancias químicas pueden provocar en las personas expuestas, se han definido diferentes tipos de valores límite umbral o TLV por sus siglas en inglés (Threshold Limit Values), mismos que se explican a continuación (Linaza, 2004)

- TLV-TWA (Valor límite umbral-Media ponderada en el tiempo), es la concentración, como media ponderada temporal, durante una jornada laboral de ocho horas (40 horas a la semana) a la cual pueden estar expuestos de manera repetida los trabajadores sin sufrir efectos adversos.

- TLV - STEL (Valor límite umbral - Límite de exposición a corto plazo), es la exposición media ponderada en un tiempo de 15 minutos, que no se debe sobrepasar en ningún momento de la jornada laboral, aun cuando la media ponderada en el tiempo que corresponda a ocho horas sea inferior al TLV-TWA. Las exposiciones por encima del TLV-TWA hasta el valor STEL no deben tener una duración superior a 15 minutos ni repetirse más de cuatro veces al día. Debe haber por lo menos un período de 60 minutos entre exposiciones sucesivas de este rango. No es un límite de exposición independiente, sino que más bien completa al TLV-TWA cuando se admite la existencia de efectos agudos de una sustancia cuyos efectos tóxicos son, principalmente, de carácter crónico. 
- TVL - C (Valor Límite Umbral-Techo), es la concentración que nunca se debe sobrepasar ni siquiera de forma instantánea,

\section{Resultados y discusión.}

En nuestro país no existen publicaciones acerca de estudios toxicológicos de Higiene Industrial para el ácido acético. Lo publicado en el exterior es poco, aunque data de antes del año 2000 y unos pocos son posteriores (Ramirez, 2011).

En el aire, la concentración de un químico se mide en partes por millón ( $\mathrm{ppm}$ ) o en peso por metro cúbico $\left(\mathrm{mg} / \mathrm{m}^{3}\right)$ (Ramirez, 2011),para el efecto se detallan los resultados recogidos en PPM en comparación con sus TLVs enlas siguientes actividades:

- Trasvase de ácido acético

- Proceso de desincrustación

Trasvase del ácido acético.

Se evalúa la tarea de trasvase del Ácido Acético tarea que realiza una vez al día y tiene una duración aproximada de 8 minutos conforme a la Figura 1.

Figura 1. Proceso de trasvase de ácido acético

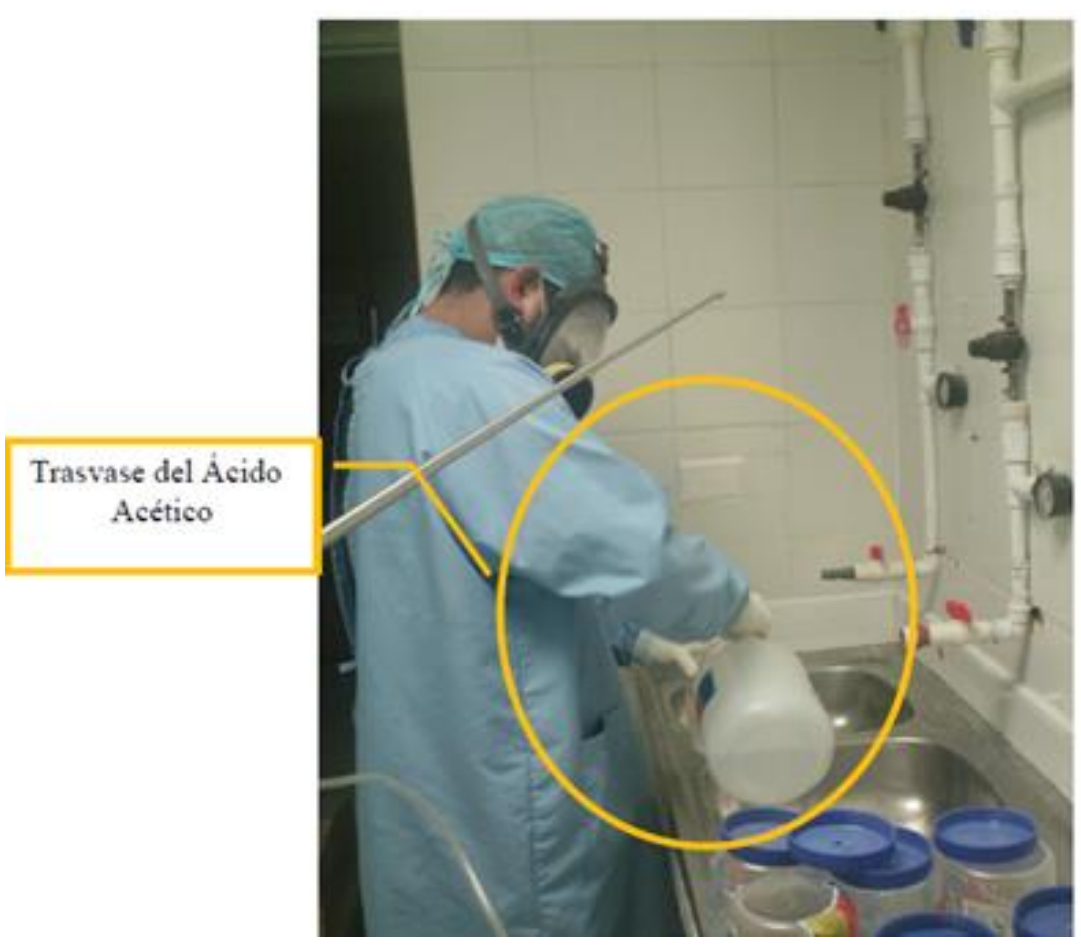

Fuente: Equipo de Investigacioón 
Los resultados obtenidos debidos de la medición de vapores de ácido acético en el proceso de trasvase, se muestran en la Tabla 1.

Tabla 1 Concentraciones de ácido acético en trasvase.

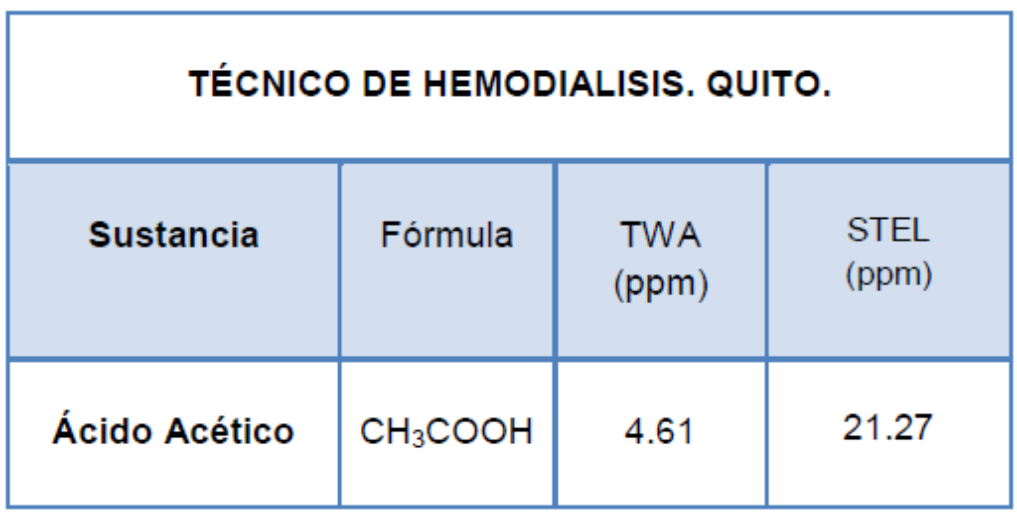

Fuente: Equipo de Investigación

Los niveles de concentración de ácido acético vulneran el valor de TLV STEL (Tabla 2) el mismo que no debe ser sobrepasado en ningún momento de la jornada laboral, constituyéndose en un riesgo intolerable para el personal operativo,

Tabla 2 Concentraciones de ácido acético en trasvase comparadas con los TLVs

\begin{tabular}{|c|c|c|c|c|c|}
\hline \multicolumn{7}{|c|}{ TÉCNICO DE HEMOdIÁLISIS. QUITO. } \\
\hline Sustancia & Fórmula & $\begin{array}{c}\text { TWA } \\
\text { Medido } \\
\mathrm{ppm}\end{array}$ & $\begin{array}{c}\text { TLV -TWA } \\
\mathrm{ppm}\end{array}$ & $\begin{array}{c}\text { STEL } \\
\text { Medido } \\
\mathrm{ppm}\end{array}$ & $\begin{array}{c}\text { TLV - } \\
\text { STEL ppm }\end{array}$ \\
\hline Ácido Acetico & $\mathrm{CH}_{3} \mathrm{COOH}$ & 4,61 & 10 & 21.27 & 15 \\
\hline
\end{tabular}

Fuente: Equipo de Investigación 
Proceso de desincrustación.

Se evalúa el proceso desincrustación de diferentes modelos de máquinas (NIPRO y NIKKISO).

De acuerdo a los procedimientos establecidos en las máquinas Nikkiso se cambia el ácido acético cada dos o tres días.

El proceso de limpieza de la máquina dura aproximadamente treinta minutos (30 min.). El Técnico de hemodiálisis coloca 16 frascos de cristal con ácido acético $500 \mathrm{ml}$., de uno en uno en cada máquina, luego coloca las mangueras en los diferentes frascos. (Figura 2).

Tiempos posteriores:

- Lavar la maquina con agua (10 min.).

- Absorción del ácido acético (20 min.).

- Tiempo de reposo (5 min.).

- Final enjuague (15 min.).

- Apagado automático.

- Veinte máquinas por noche sin incluir las máquinas de hepatitis.

Figura 2.Proceso de limpieza de máquinas de hemodiálisis.

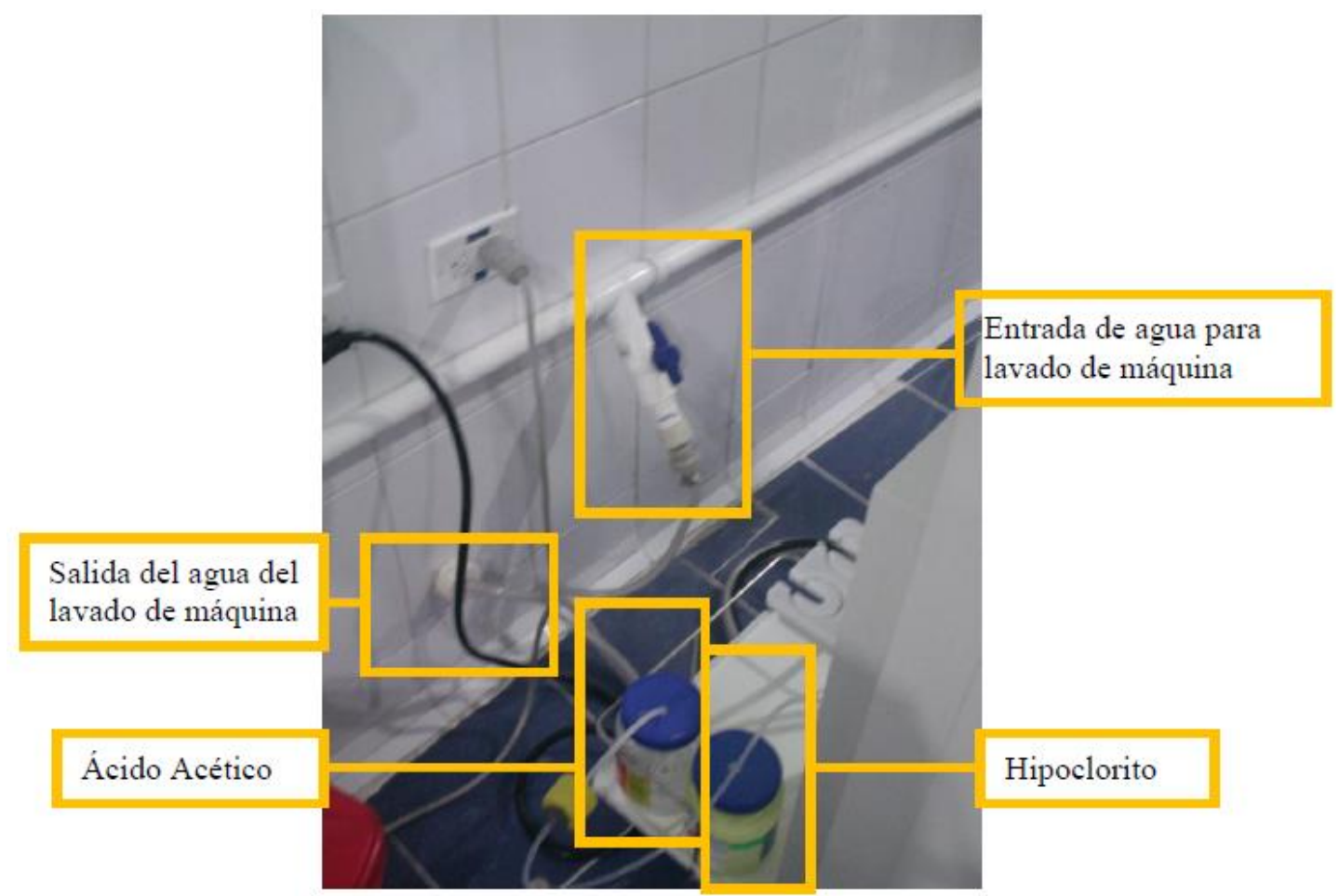

Fuente: Equipo de Investigación 
Los resultados de la medición de vapores de ácido acético en el proceso de desincrustación se muestran en la Tabla 3

Tabla 3. Concentraciones de ácido acético en la desincrustación

\begin{tabular}{|l|c|c|c|}
\hline \multicolumn{3}{|c|}{ PROCESO DE DESINCRUSTACION } \\
\hline Sustancia & Fórmula & $\begin{array}{c}\text { TWA } \\
(\mathrm{ppm})\end{array}$ & $\begin{array}{r}\text { STEL } \\
(\mathrm{ppm})\end{array}$ \\
\hline Ácido Acético & $\mathrm{CH}_{3} \mathrm{COOH}$ & 4.22 & 13.55 \\
\hline
\end{tabular}

Fuente: Equipo de Investigación

En este proceso la concentración de ácido acético se encuentra por debajo de los límites de exposición permitida conforme la comparación con los TLVs (Tabla 3)

Tabla 3. Concentraciones de ácido acético en la desincrustación comparadas con los TLVs

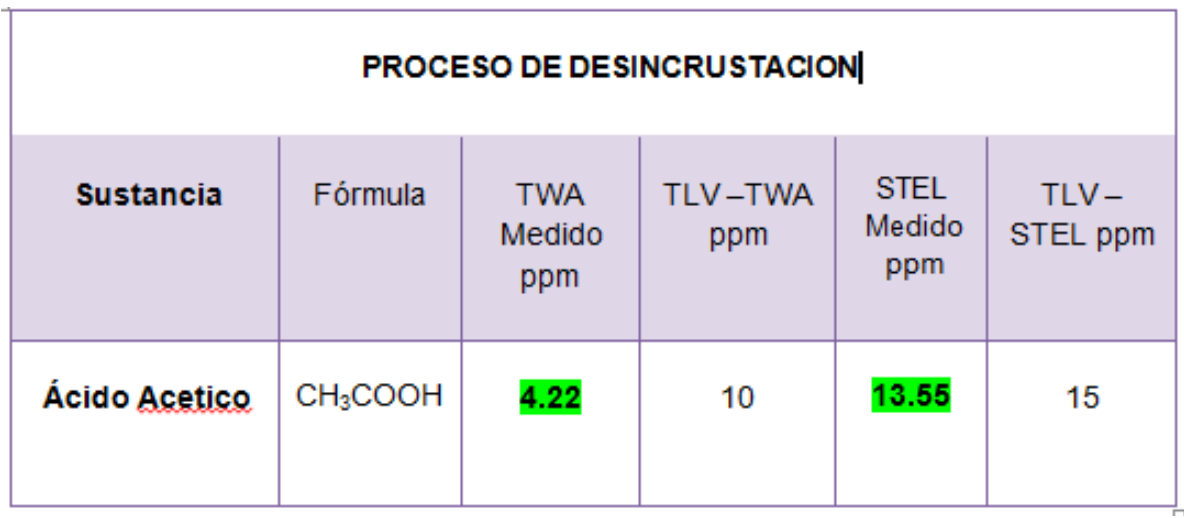

Fuente: Equipo de Investigación

La revisión periódica de las conexiones de las botellas y de la instalación de gases en su caso, es la medida preventiva más eficaz para la prevención de fugas que puedan ser causa de una situación de emergencia (Solá, 2017). 


\section{Conclusiones •}

- Las mediciones en la concentración del ácido acético en el proceso de trasvase no supera el TLV-TWA.

- La medición de la concentración del ácido acético en el proceso de trasvase en el caso del TLV-PPM STEEL si excede la concentración permitida.

- El proceso de desincrstación, no genera ningún riesgo a la salud de los colaboradores expuestos al ácido acético, debido a que su concentración, comparada con los TLVs, no sobrepasan los valores permisibles en el ambiente.

- La Empresa debe reducir los efectos de la exposición a agentes químicos a través de la dotación de EPP, la aplicación del Plan de Vigilancia a la Salud.

\section{Referencias bibliográficas.}

Asturias, F. M. (2000). Manual básico de prevención de riesgos laborales: higiene industrial, seguridad y ergonomía. Edita: Sociedad Asturiana de Medicina y Seguridad en el Trabajo. Madrid, Año.

Cabañas, I. M. (2001). Prevención del riesgo químico en los laboratorios de docencia e investigación. In Anales de la Real Sociedad Española de Química (No. 2, pp. 60-66). Real Sociedad Española de Química.

Calera, R,A,, Roel, V,M, Casal, L,A, Gadea, M,R,, Cencillo, F,R, Riesgo Químico Laboral: Elementos para un diagnóstico en España, Rev, Esp, Salud Pública; 79(2): 120-35, (2005)

Castro Delgado, R., \& Arcos González, P. (1998). El riesgo de desastre químico como cuestión de salud pública. Revista española de salud pública, 72, 481-500.

Cújar-Vertel, A. D. C., \& Julio-Espitia, G. P. (2016). Evaluación de las condiciones térmicas ambientales del área de producción en una panadería en Cereté (Córdoba). Entramado, 12(1), 332-343.

Henschler D. (1984): "Exposure Limits: History, Philosophy, Future Developments". Ann. Occup. Hyg.; 28 (1): 79-92.

Izmerov N. (1973): Control Air Pollution in the USSR. Public Health Paper No. 54. WHO. Geneva. 
Linaza, L. M. A. (2004). Guía para la implantación de un sistema de prevención de riesgos laborales. FC Editorial.

Mangosio, J. (2008). Higiene y seguridad en el trabajo. Buenos Aires-Argentina. Cámara Argentina del Libro.

Paolucci, V., Romeo, R., Sisinni, A. G., Bartoli, D., Mazzei, M. A., \& Sartorelli, P. (2015). Silicosis en trabajadores expuestos a conglomerados artificiales de cuarzo: $₫$ es distinta a la silicosis crónica simple?. Archivos de Bronconeumología, 51(12), e57-e60.

Paustenbach D, y Langner R. (1986): "Corporate Occupational Exposure Limits: the current state of affairs”. Am. Ind. Hyg. Assoc. J.; 47 (2): 809-818.

Ramírez, A. V. (2011, January). Exposición toxicológica en las grandes alturas:¿ es necesario corregir los valores límite umbral de exposición de tóxicos?. In Anales de la Facultad de Medicina(Vol. 72, No. 1, pp. 61-67). UNMSM. Facultad de Medicina.

Ramírez, A. V. (2011, July). Mejora de los indicadores biológicos de exposición al mercurio en trabajadores de una refinería de oro. In Anales de la Facultad de Medicina (Vol. 72, No. 3, pp. 177-182). UNMSM. Facultad de Medicina.

Solá, X. G., Carrera, E. G., \& Farrás, (2017) M. R. NTP 399: Seguridad en el laboratorio: actuación en caso de fugas y vertidos.

Yanes, L. (2002). Límites de Exposición Ocupacional y Ambiental: una visión crítica. Salud de 1

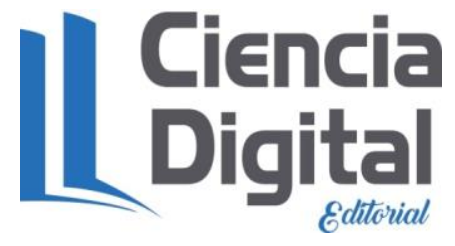




\section{Para citar el artículo indexado.}

Laverde C., Torres R., Simancas J., Perez A. \& Guaman C. . (2018). Medición de niveles de ácido acético en el proceso de limpieza de máquinas Hemodialíticas en una unidad de tratamiento renal. Revista electrónica Ciencia Digital 2(3), 288-298. Recuperado desde: http://cienciadigital.org/revistacienciadigital2/index.php/CienciaDigital/article/view/153/13 $\underline{8}$

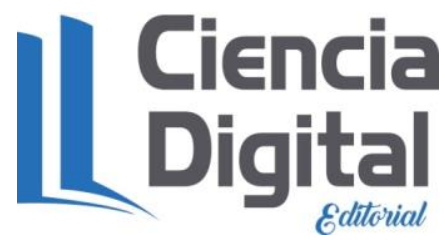

El artículo que se publica es de exclusiva responsabilidad de los autores y no necesariamente reflejan el pensamiento de la Revista Ciencia Digital.

El articulo queda en propiedad de la revista y, por tanto, su publicación parcial y/o total en otro medio tiene que ser autorizado por el director de la Revista Ciencia Digital.
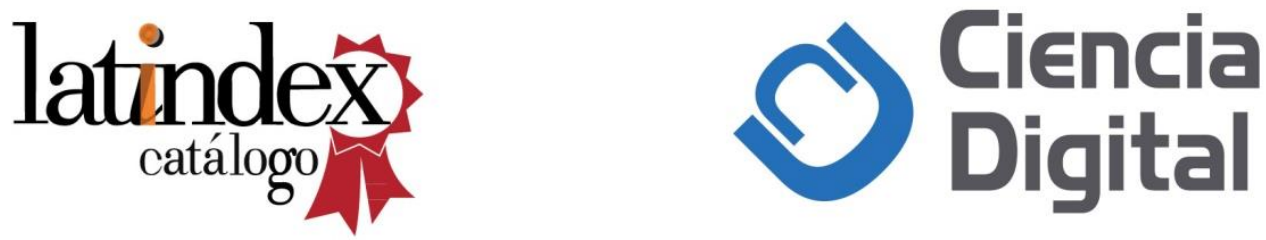